\title{
Effects of familiarity on preschool children's recall
}

\author{
CHRISTINE M. TODD and MARION PERLMUTTER \\ Institute of Child Development, University of Minnesota, Minneapolis, Minnesota
}

\begin{abstract}
Effects of prior interaction with stimuli on preschool children's free recall was examined. Half of the children in each of two age groups ( 3 years and 5 years) were given 6 items to play with for 5 days prior to free recall testing of those items plus 6 new items. The remaining subjects had no prior experience with any of the stimuli on the 12-item test list. Effects of prior interaction with stimuli were obtained for females in both age groups. Significant clustering based on play category was observed. In addition, prior interaction increased recall of play items and decreased recall of nonplay items.
\end{abstract}

The interplay between knowledge and memory performance has received much attention in recent years. Typically, semantic knowledge, that is, the abstract information that forms the basis of one's conceptual system (Tulving, 1972), has been of interest. The use of this semantic knowledge during encoding and retrieval has been found to enhance performance of older children and adults in deliberate free-recall situations (e.g., Cole, Frankel, \& Sharp, 1971; Kail, 1976; Moely \& Shapiro, 1971).

The use of semantic knowledge to facilitate recall of preschool children is less clear. Although research has indicated that even 2-year-olds benefit from semantically organized lists (Goldberg, Perlmutter, \& Myers, 1974) and that 3-year-olds benefit from blocked presentation and semantic category cues, the effect of these semantic manipulations has been found to be greater for older than for younger children (e.g., Furth \& Milgram, 1973; Laurence, 1967; Perlmutter \& Myers, 1979; Perlmutter \& Ricks, 1979). Such findings should not be surprising, given that very young children still are in the process of forming conceptual and semantic categories. The semantic system, however, is not the only knowledge that is potentially available to young children. For example, Tulving (1972) identified episodic knowledge as a record of specific past experiences that are localized in time and/or space. Children as young as 3 years old have been shown to be relatively proficient in recalling specific information about naturally occurring past events (Todd \& Perlmutter, 1980). Perhaps this episodic knowledge plays a more important role prior to the time that semantic knowledge is fully operational.

The influence of episodic knowledge on memory may

This research was supported by grants from NICHHD (11776 and 01136$)$ and from NSF (22075). We would also like to thank colleagues at the Institute of Child Development for their critical reading of the manuscripts. Requests for reprints should be sent to Marion Perlmutter, Institute of Child Development, 51 East River Road, University of Minnesota, Minneapolis, Minnesota 55455 . not be evident in most memory studies, since experimental tasks typically involve rapid sequential presentation of single items. Little episodic information about the stimuli, except the sequential order of presentation is likely to be available. Simultaneous presentation of items affords a somewhat greater opportunity for the establishment of rich episodic traces, especially if subjects are allowed to play with the items. However, this procedure has been used in only a few studies, and the results have been mixed, showing better levels of recall for some age groups but not for others (Furth \& Milgram, 1973; Perlmutter \& Ricks, 1979).

The purpose of the present research was to assess the effects of prior interaction with stimuli on subsequent recall by 3- and 5-year-olds. Children were allowed to play with a group of toys before administration of a standard free-recall task. It was expected that prior interaction with the stimuli would establish episodic traces that could be used during encoding and retrieval of the subsequent memory task.

\section{METHOD}

\section{Subjects}

Forty 3 -year-olds (mean $=3$ years 2 months; range $=3$ years 0 months to 3 years 5 months) and 405 -year-olds (mean = 5 years 0 months; range $=4$ years 10 months to 5 years 5 months) participated in this study. Equal numbers of males and females in each age group were assigned randomly to the experimental and control groups.

\section{Materials}

Six items that could be named easily by young children were the stimuli. They were cardboard three-dimensional colored objects obtained from Whitman Tiny Activity Books. Two sixitem sets (Set A-train, truck, tractor, lion, cow, rooster; Set Bfire engine, car, airplane, elephant, horse, sheep) were constructed by randomly combining three items from each of the two conceptual categories (animals and vehicles), with the restriction that the two sets contained items of approximately equal size.

\section{Procedure}

For the experimental group, one of the six-item sets was 
mailed to the family of each subject. The parents were instructed to present the items to their children once each day for up to $30 \mathrm{~min}$ on 5 consecutive days prior to testing, and were sent forms on which to record the amount of time their child played with the toys. The two sets of toys were counterbalanced across subjects so that half of the children played with Set A and half with Set B.

On the day following the last play session, the children were tested individually by a female experimenter. The experimenter presented each item in the set by individually showing it, asking the child to label it, and replacing it in a box at a rate of approximately one every $10 \mathrm{sec}$. The standard order of presentation was random, with the restriction that no more than two consecutive items were from a single semantic or play category. Following presentation of the last item, the box was covered and the child was tested for recall.

\section{RESULTS}

\section{Prior Interaction With Stimuli}

The data provided by the parents of children in the experimental group on the amount of time that their children interacted with the toys prior to testing is shown in Figure 1. Over the five days, the children played with the toys for an average of $67 \mathrm{~min}$. A 2 (age) x 2 (sex) x 2 (set) x 5 (days) analysis of variance indicated that the amount of time spent playing with toys decreased across days $[\mathrm{F}(4,128)=13.55, \mathrm{p}<.01]$. In addition, Set $B$ was played with significantly longer than Set A $[F(1,32)=7.04, p<.05]$. There were no significant main effects of age or sex and no significant interactions.

\section{Evidence for Episodic Traces Resulting From Prior Interaction}

During presentation of the test items, $80 \%$ of the children in the experimental group at each age level made at least one comment concerning their prior interaction with the toys at home; and many children commented on their prior interactions with each of the play items. No such comments were made concerning new items, and none of the children in the control condition indicated familiarity with any of the stimuli. Thus, it appeared that the children in the experimental group

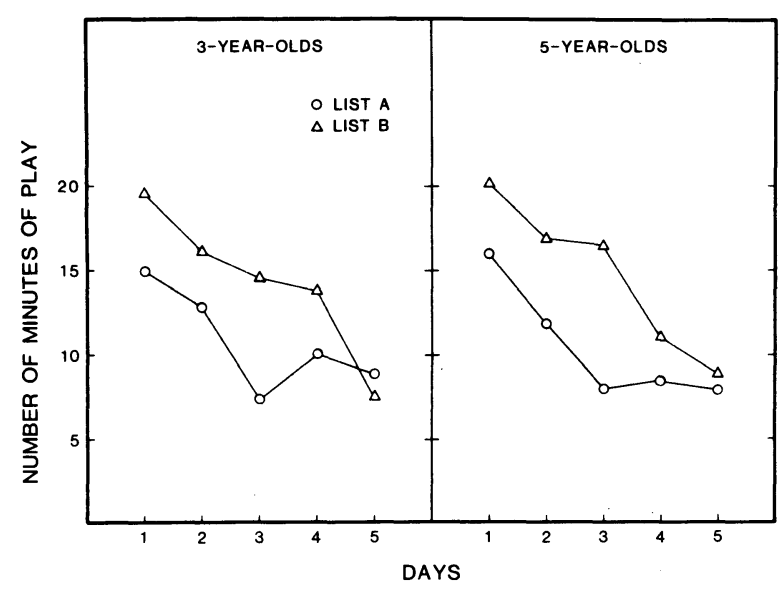

Figure 1. Amount of home interaction prior to testing.

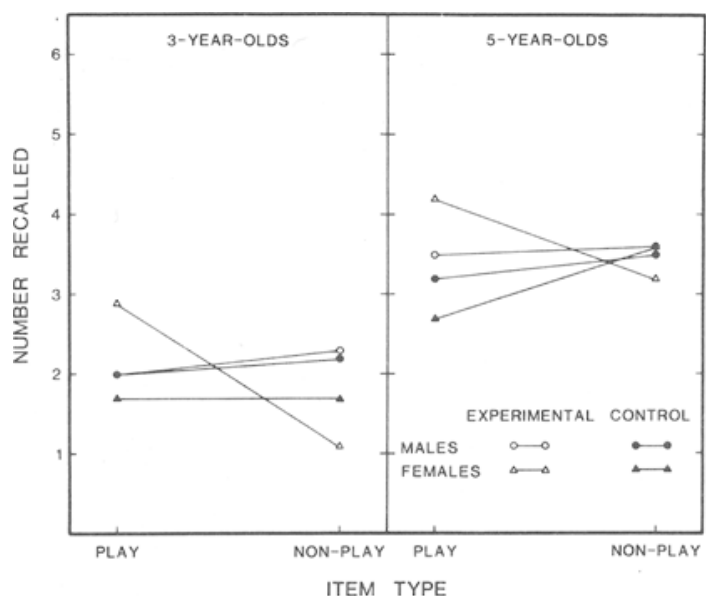

Figure 2. Number of play and nonplay items recalled by each sex in each condition at each age.

had established episodic traces through interaction with the stimuli, and that these traces were accessed during presentation of the items in the laboratory.

\section{Level of Recall}

The mean level of play and nonplay items recalled by each sex, in each age group, in each condition, is shown in Figure 2. A 2(condition) x 2(age) x 2(sex) x 2(set) $x$ 2(item type) analysis of variance was performed on these data. It should be noted that, for the control group, assignment of item type (play, nonplay) was random and was simply carried out as a control.

Five-year-olds recalled significantly more than did 3-year-olds (6.87 and 3.97 items, respectively) $[F(1,64)$ $=62.15, \mathrm{p}<.01]$, but age did not interact with any other variables. Likewise, none of the other main effects approached statistical significance. However, the condition $x$ item type $[F(1,64)=6.64, p<.05]$, condition $x$ sex $x$ item type $[F(1,64)=5.96, p<.05]$, and set $x$ item type $[F(1,64)=15.46, p<.01]$ interactions were statistically significant. As may be seen in Figure 2, both younger and older females in the experimental group recalled significantly more play than nonplay items (3.55 vs. 2.15 , respectively) $[\mathrm{t}(19)=2.93, \mathrm{p}<.01]$ and more play items than did females in the control condition ( 3.55 vs. 2.20 , respectively) $[\mathrm{t}(38)=2.94, \mathrm{p}<.01]$. However, this effect was not observed for males in the experimental group. In addition, as would be expected, recall of play and nonplay items did not differ for any of the control subjects. The set $\mathrm{x}$ item type interaction was the result of children's recalling significantly more play than nonplay items from Set B, which was interacted with most at home (3.12 and 2.28 items, respectively) $[\mathrm{t}(30)=3.13, \mathrm{p}<.01]$, and significantly more nonplay than play items from Set A (3.02 and 2.42 items, respectively) $[\mathrm{t}(30)=-2.08, \mathrm{p}<.05]$.

\section{Semantic Clustering}

Semantic clustering was computed using the standard $\mathrm{RR}$ clustering index. A 2(condition) $\times 2$ (age) $\times 2$ (sex) 
x 2 (set) analysis of variance indicated a marginally significant age difference $(.49$ vs. .59 , respectively) $[\mathrm{F}(1,64)$ $=3.09, \mathrm{p}<.10]$. No other main effects or interactions approached significance.

\section{Play Clustering}

The following formula was used to assess play clustering: (number of successive repetitions of play items)/ (total number of play items recalled minus one). A 2(condition) $\times 2$ (age) $\times 2$ (sex) $\times 2$ (set) analysis of variance indicated a significant main effect of condition $[F(1,64)=$ 9.33, $\mathrm{p}<.01]$ and a significant condition $\mathrm{x}$ sex interaction $[F(1,64)=3.99, p<.05]$. Although clustering scores of males in the experimental group were somewhat higher than those of males in the control group (.51 and .41, respectively), the difference in play clustering for experimental and control subjects was significant only for the females (.62 and .17 , respectively) [t(38) $=3.91, \mathrm{p}<.01]$.

\section{Reaction Time to Recall First Item}

There appeared to be differential speed in recall for the experimental and control groups. To investigate this possible difference, time to recall of the first item was obtained from the audiotapes. Five children in the control group and one child in the experimental group were omitted from this analysis due to poor tape quality, as were two children in the control group who were unable to recall any items. A 2 (condition) $\times 2$ (age) $\times 2$ (sex) $\times$ 2 (set) analysis of variance indicated that children in the experimental condition took significantly longer to recall the first item than did children in the control condition $(20.90$ and $7.44 \mathrm{sec}$, respectively) $[\mathrm{F}(1,56)=$ $4.17, \mathrm{p}<.05]$ and that females took significantly longer than males $(20.82$ and 8.64 , respectively) $[\mathrm{F}(1,56)=$ $4.39, \mathrm{p}<.05]$. There were no other significant main effects or interactions. For females in the experimental group, this delay appeared to be associated with initial recall of play items. Of the first two items recalled by these subjects, $70 \%$ were play items, as compared with only $41 \%$ for females in the control group.

\section{DISCUSSION}

The purpose of this experiment was to assess the effects of prior interaction with stimuli on preschool children's subsequent recall. It was hypothesized that episodic knowledge may play an important role in recall prior to the age when children's semantic knowledge is well developed. Young children may search through episodic "categories" in much the same way that older children and adults are presumed to search through semantic categories, or simple association between items in episodic memory may lead to improvement in recall. The results indicated that episodic knowledge about the items was established during the children's prior interaction with the stimuli. During presentation of the memory task, the children indicated their recognition of the toys. Moreover, some of the children apparently used this knowledge during recall, as evidenced by the enhanced recall of play items obtained for females.

It is unclear why effects of prior play were restricted almost entirely to females, and why enhanced recall of play items was paired with depressed recall of nonplay items. However, the reaction time data suggests one possible explanation. Females in the experimental group took over three times as long to recall the first item as the other three groups. Furthermore, in recall of the first two items, their retrieval of play items was over twice that of nonplay items. It appears that females who had prior experience with some of the stimuli tended to search and report their recall of these items first. If these play items were stored in long-term memory, the prolonged memory search for play items by females in the experimental group may have resulted in the loss of information about nonplay items in short-term memory. Thus, although prior interaction with stimuli may have enabled the females to recall more information from long-term memory, the resulting benefit may have been offset by a corresponding loss of information from short-term memory.

In summary, there is some indication that very young children use episodic information to aid retrieval. Thus, it appears that both episodic and semantic knowledge aid recall in early childhood; however, neither of these knowledge supports seems sufficient to evoke levels of recall observed in older children. Moreover, although use of semantic knowledge is known to increase with age, thus far there is no evidence that use of episodic knowledge changes with age.

\section{REFERENCES}

Cole, M., Frankel, R., \& Sharp, D. Development of free recall learning in children. Developmental Psychology, 1971, 4, 109-123.

Furth, H. G., \& Milgram, N. A. Labeling and grouping effects in recall of pictures by children. Child Development, 1973, 44, 511-518.

Goldberg, S., Perlmutter, M., \& Myers, N. Recall of related and unrelated lists by 2-year-olds. Journal of Experimental Child Psychology, 1974, 18, 1-8.

KAIL, R. B. Children's encoding of taxonomic classes and subclasses. Developmental Psychology, 1976, 12, 487-488.

Laurence, M. W. A developmental look at the usefulness of list categorization as an aid to free recall. Canadian Journal of Psychology, 1967, 21, 153-165.

Moely, B. E., \& Shapiro, S. I. Free recall and clustering at four age levels: Effects of learning to learn and presentation method. Developmental Psychology, 1971, 4, 490.

Perlmutter, M., \& Myers, N. A. Recall development in twoto-four-year-olds. Developmental Psychology, 1979, 15, 73-83.

Perlmutter, M., \& Ricks, M. Recall in preschool children. Journal of Experimental Child Psychology, 1979, 27, 423-436.

Todd, C. M., \& Perlmutter, M. Reality recalled by preschool children. In M. Perlmutter (Ed.), New directions for child development: Children's memory. San Francisco: Jossey-Bass, 1980.

Tulving, E. Episodic and semantic memory. In E. Tulving \& W. Donaldson (Eds.), Organization of memory. New York: Academic Press, 1972.

(Manuscript received for publication March 24, 1983.) 